\title{
A Rare Cause Of Hypertension In Pregnancy
}

\section{Harborow CE ${ }^{1 *}$, Waghorn AJ ${ }^{2}$, Davison AS 1,3}

Departments of Clinical Biochemistry and Metabolic Medicine, Liverpool Clinical Laboratories' ${ }^{1}$, Endocrine and Breast Surgery², Royal Liverpool and Broadgreen University Hospitals Trust, Liverpool, UK; Manchester Academic Health Science Centre ${ }^{3}$, University of Manchester, UK ${ }^{3}$

\section{Case Description}

A pregnant (13/40) 23-year-old female with severe resistant hypertension was referred to our specialist unit. BP on arrival was $240 / 140 \mathrm{mmHg}$ after one month of Labetalol $(200 \mathrm{mg}$ tds). Clinical symptoms included headaches, migraines and flushing for several years, especially after eating, and worse during pregnancy. She had pre-eclampsia in her first pregnancy, diagnosed at 20 weeks, with hypertension and proteinuria. She was untreated and no repeat BP was recorded between her first child's birth and this pregnancy.

Urine metadrenalines showed a high urine NMN (Table 1) and MRI of the abdomen and pelvis (Figure 1) revealed a $4.2 \times 3.1 \times 3.5 \mathrm{~cm}$ lesion on the left adrenal gland suggestive of a phaeochromocytoma (PCC).

\section{Medical management}

Labetalol was stopped and phenoxybenzamine (10 mg tds) prescribed. Propranolo $(10 \mathrm{mg})$ was also administered as required, when heart rate exceeded $100 \mathrm{bpm}$ lying down. Plasma metadrenalines prior (off medications) revealed elevated NMN and 3-MT concentrations (Table 1). Other than mildly raised white cells (15.6 x $10 \%$ L, RR 3.5-11.0), all other investigations (including parathyroid hormone, calcitonin, chromogranin A and gastrin) were unremarkable. An ultrasound of the neck showed no abnormalities suggestive of a paraganglioma.

\section{Surgery}

Prior to surgery phenoxybenzamine was increased gradually from $10 \mathrm{mg}$ tds to $40 \mathrm{mg}$ qds; propranolol was also increased to $30 \mathrm{mg}$ qds. After adequate $\alpha$ - and $\beta$ blockade, a left laparoscopic adrenalectomy was performed (at 15/40). The patient's BP stabilised to $118 / 65 \mathrm{mmHg}$ and she was discharged four days later. At discharge plasma metadrenaline concentrations had normalised (Table 1).

Histological analysis revealed an encapsulated neoplasm showing infiltration of the capsule and possible minimal infiltration of periadrenal fat. No lymphovascular invasion was noted. The tumour had a Phaeochromocytoma of the adrenal gland scaled score (PASS) of 3 (maximum score possible 20). Immunohistochemistry revealed that the cells expressed strong diffuse positivity for chromogranin and synaptophysin; thus confirming a PCC. No significant features to suggest a potential malignancy were observed.

\section{Follow up}

Planned annual plasma metadrenaline concentrations with imaging if appropriate. Genetic evaluation of RET, VHL, SDHB and SDHD genes; mutations which are linked to hereditary conditions such as multiple endocrine neoplasia type-2, Von Hippel-Lindau disease and familial paraganglioma and PCC, respectively. Family history revealed an older sister had a PCC removed 14 years previously aged 12 suggesting family members should also be offered testing for familial PCC. Genetic testing was not considered at that time as it was not widely available.

\section{POINTS TO CONSIDER}

PCC should always be considered in the differential diagnosis of hypertension during pregnancy, especially when presentation is before 20 weeks and when patients are resistant to pharmacological treatment.

Biochemical evaluation of PCC during pregnancy is essential as the use of diagnostic radiology if often limited. It is therefore vital that patients have their diet and or medications optimised prior to testing.

Genetic testing of a pregnant patient is highly important in evaluating the familial basis of PCC and the future risk of malignancy.

A multidisciplinary approach involving biochemistry, radiology, obstetrics, anaesthetics, and surgery colleagues is important in cases of PCC in pregnancy due to the unique difficulties in diagnosis and management of both mother and baby.

\begin{tabular}{|l|c|c|c|}
\hline Analyte & A & B & Reference Range \\
\hline Urine Normetadrenaline & 53.4 & - & $<4.4 \mu \mathrm{mol} / 24 \mathrm{~h}$ \\
\hline Urine Metadrenaline & 0.4 & - & $<2.0 \mu \mathrm{mol} / 24 \mathrm{~h}$ \\
\hline Plasma Normetadrenaline & 15070 & 780 & $<1180 \mathrm{pmol} / \mathrm{L}$ \\
\hline Plasma Metadrenaline & 96 & 143 & $<510 \mathrm{pmol} / \mathrm{L}$ \\
\hline Plasma 3-methoxytyramine & 343 & $<75$ & $<180 \mathrm{pmol} / \mathrm{L}$
\end{tabular}

Table 1. Metadrenaline results. (A) Initial measurement; (B) Follow-up measurement post surgery. Plasma and urine metadrenalines were measured using liquid chromatography tandem mass spectrometry. Plasma samples were collected in a seated position after 20mins rest. Reference ranges are based on a seated reference population.

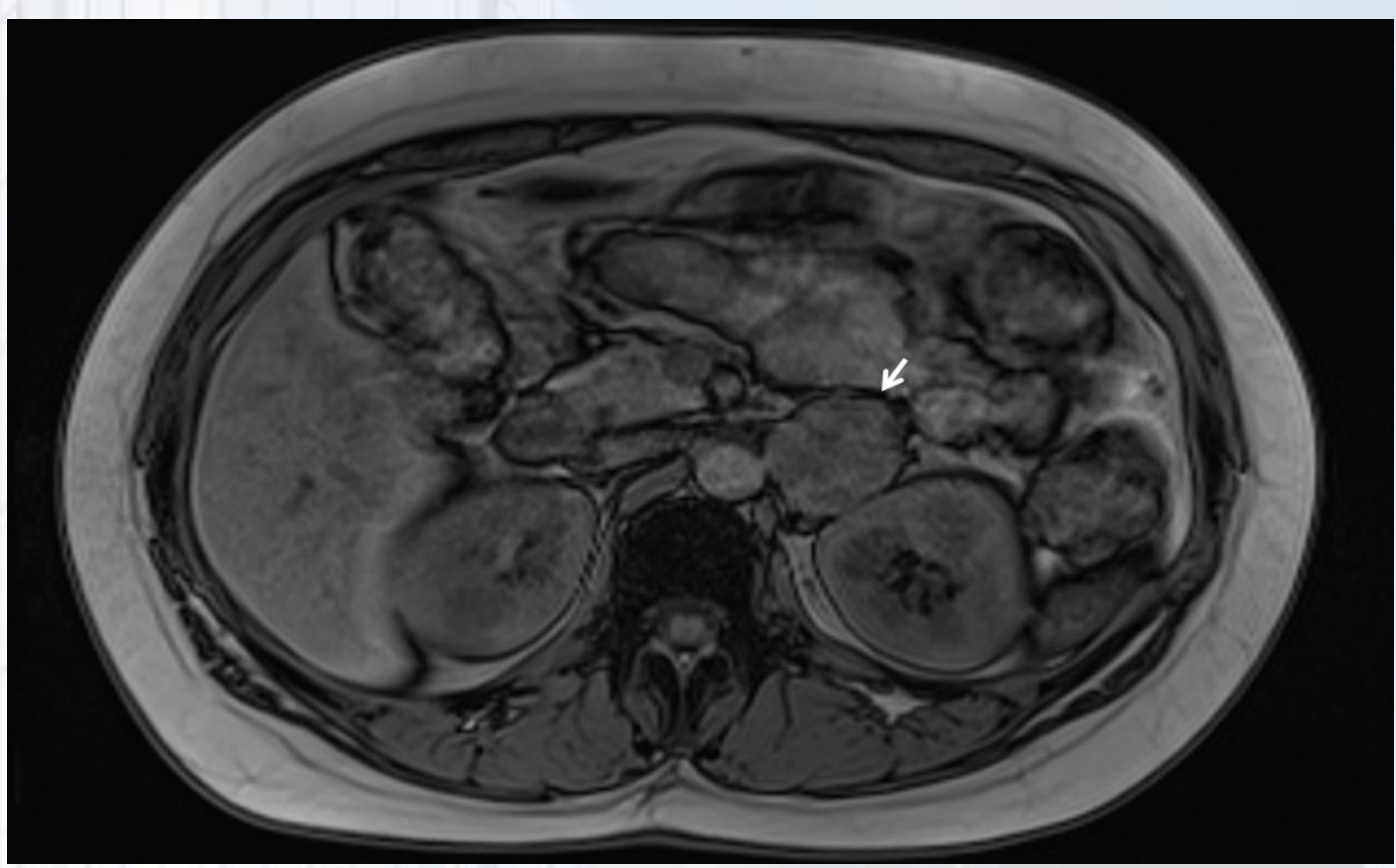

Figure 1. Axial magnetic resonance image of the abdomen showing a $4.2 \times 3.1 \times 3.5 \mathrm{~cm}$ lesion on the left adrenal gland (arrow).

\section{Discussion}

Diagnosis: Endocrine Society Clinical Practice Guideline for PPGL recommends:

- Biochemical testing for PCC should include measurement of plasma free or urinary fractionated metadrenalines, using mass spectrometry or electrochemical detection.

Drugs known to cause a physiological increase in metadrenalines should be excluded where possible before measurement of metadrenalines.

- MIBG and CT are contraindicated in pregnancy but MRI can be used safely.

- Pregnant women should be evaluated and treated by multidisciplinary teams at centres with appropriate expertise.

Treatment: The most frequently used medical management in PCC is a-blockade with phenoxybenzamine; which crosses placenta but is deemed safe to use in pregnancy. ${ }^{2}$ Use of $\beta$-blockers in pregnancy is controversial but short-term use alongside $\alpha$-blockers may be necessary to control tachycardia. Prolonged pharmacologic treatment is not well supported in the literature; delay before surgery may increase the likelihood of adverse outcomes for the foetus. ${ }^{2}$ If diagnosed during the third trimester, pharmacologic management may be preferable until the foetus is viable. ${ }^{3}$

Follow-up: Measurement of metadrenaline concentrations (plasma or urine) post-operatively. ${ }^{4}$ MIBG scintigraphy and CT are useful postpartum to ensure all tumours have been excised. Malignant, extra-adrenal and multiple tumours suggest a higher likelihood of a mutation. Age of the patient is also important; likelihood of a mutation in patients with non-syndromic PCC less than 45-yearsold is 5 -fold higher than in older patients. ${ }^{1}$ Pregnant women are generally a young patient group and so genetic testing can be justified.

Better outcomes are achieved when diagnosis of PCC is made during the antenatal period; $86 \%$ survival of both mother and baby in the antenatal period versus $57 \%$ if during labour or postpartum. ${ }^{2}$ 